\title{
Competencias Digitales en los Estudiantes del Grado de Maestro de Educación Primaria. El caso de tres Universidades Españolas
}

\author{
María A. Pascual(1), José A. Ortega-Carrillo(2), Miguel Pérez-Ferra(3) y Javier Fombona(4)* \\ (1) Facultad de Formación del Profesorado y Educación, Universidad Oviedo, Aniceto Sela s.n., Oviedo - España. \\ (e.mail: apascual@uniovi.es) \\ (2) Facultad de Ciencias de la Educación, Departamento de Didáctica y Organización Escolar, Universidad de Granada, \\ Campus Universitarios de Cartuja, s/n, Granada - España. (e.mail: jaorte@ugr.es) \\ (3) Facultad de Humanidades y Ciencias de la Educación, Departamento de Pedagogía, Universidad de Jaén, Campus \\ Las Lagunillas s/n. Jaén - España (e.mail: mperez@ujaen.es) \\ (4) Facultad de Formación del Profesorado y Educación, Universidad de Oviedo, Aniceto Sela s/n. Oviedo - España \\ (e.mail: fombona@uniovi.es)
}

* Autor a quien debe ser dirigida la correspondencia

Recibido Abr. 1, 2019; Aceptado May. 20, 2019; Versión final Jun. 21, 2019, Publicado Dic. 2019

\begin{abstract}
Resumen
Se analiza la competencia digital del alumnado del Grado de Maestro de Educación Primaria cuando cursa primer curso con el fin de diseñar un programa formativo adaptado a sus necesidades digitales. Esto se enmarca en el hecho de que los conocimientos y las habilidades de los estudiantes universitarios en la competencia digital son cruciales para un aprendizaje eficaz, motivador e inclusivo. Sin embargo, son reducidos los estudios que cuantifican el nivel de conocimientos y destrezas prácticas que realmente posee este alumnado cuando llega a los estudios superiores. El trabajo sigue una metodología descriptivaexploratoria y utiliza un cuestionario validado para analizar una muestra de 559 estudiantes, futuros docentes, de tres universidades españolas, Granada, Jaén y Oviedo. Los resultados detallan cinco dimensiones: información, comunicación, creación de contenidos, seguridad, y resolución de problemas. Se concluye que hay desconocimiento en la forma adecuada de gestionar la información; en la comunicación y seguimiento de la huella digital y carencias sobre cómo resolver nuevos problemas informáticos.
\end{abstract}

Palabras clave: competencia digital; educación superior, maestro, formación inicial del profesorado

\section{Digital Competences in the Students of Degree Primary Education Teacher}

\begin{abstract}
This research is carried out in 2018, and it analyses this digital competence of the Primary Education Master Degree students in the first academic year, in order to design a training program adapted to their digital needs. This study is based on the fact that college students' knowledge and skills in digital competence are important to face an effective, motivating and inclusive learning. However, there are few studies to quantify the level of knowledge and practical skills of these students in higher education. This work follows a descriptive-exploratory methodology and it uses a validated questionnaire to analyze a sample of 559 students, future teachers, from three Spanish universities, Granada, Jaén and Oviedo. The result differentiates five dimensions and levels: information, communication, content creation, security, and problem solving. It is concluded that there exists lack of knowledge about the proper way to manage information, about the communication and monitoring of the fingerprint, and deficiencies on how to solve new computer problems.
\end{abstract}

Keywords: digital competence; learning; higher education, teacher, initial teacher training 


\section{INTRODUCCIÓN}

En los últimos años, el Espacio Europeo de Educación Superior ha impulsado un modelo didáctico adaptado a los cambios, con el foco de atención en el estudiante, sus contextos y centrado en el desarrollo de competencias (Baartman et al., 2007). Esto se ha traducido en una mayor adecuación de las dinámicas educativas a las características del entorno, y más específicamente, a los requisitos tecnológicos. La formulación del sistema europeo de transferencia de créditos entre titulaciones universitarias europeas, ECTS, a través de competencias, analizada en el Proyecto Europeo Tuning, supone la descripción concreta de los resultados del aprendizaje de un determinado programa. Pero el término competencia es más que conocimientos y habilidades, como se señala en el Proyecto DeSeCo ya que involucra la capacidad de afrontar tareas problemáticas mediante el uso de recursos psicosociales en diferentes tipos de contexto Por lo que el término competencia adopta un enfoque holístico, sociocultural y constructivista.

Las competencias transversales o genéricas son consideradas fundamentalmente conocimientos, habilidades, actitudes y valores que despliegan los estudiantes para movilizar la adquisición de los aprendizajes específicos de la titulación que cursan (Strijbos, et al., 2015), y son transferibles al saber estar y al saber ser y, por consiguiente, a situaciones y acciones concretas. En el mismo sentido, este conocimiento debe enriquecer al estudiante como persona, y aportarle recursos y habilidades para el dominio de las competencias específicas para la interacción con el entorno. Este escenario está marcado por la evolución y dinamismos de una sociedad altamente tecnológica. Así, ya en 2007 la Comisión Europea destaca la competencia digital como: el uso seguro y crítico de las TIC para el trabajo, el ocio y la comunicación. Se sustenta en las competencias básicas en materia TIC: el uso del ordenador para obtener, evaluar, almacenar, producir, presentar e intercambiar información y comunicarse y participar en redes de colaboración a través de Internet.

La competencia digital es por tanto un pre-requisito interdisciplinar para que los estudiantes de todas las edades puedan beneficiarse por completo de las nuevas posibilidades que ofrece la tecnología para un aprendizaje más eficaz, motivador e inclusivo (Comisión Europea, 2013). Y así queda reflejado en España en la Orden ECD/65/2015 indicando que: la competencia digital es aquella que implica el uso creativo, crítico y seguro de las TIC para alcanzar los objetivos relacionados con el trabajo, la empleabilidad, el aprendizaje, el uso del tiempo libre, la inclusión y participación en la sociedad. Esta competencia supone, además de la adecuación a los cambios que introducen las nuevas tecnologías en la alfabetización, la lectura y la escritura, un conjunto nuevo de conocimientos, habilidades y actitudes necesarias hoy en día para ser competente en un entorno digital.

Es fundamental generar procesos educativos que favorezcan la adquisición de la competencia digital por parte de los estudiantes y diseñar esos procesos desde una perspectiva crítica de las tecnologías de la información y la comunicación (TIC) que las hagan avanzar desde una conciencia social. Como señalan Pérez el al. (2016) el desarrollo de la competencia digital no se adquiere únicamente por la presencia e interacción con los medios. En esta línea el Informe Horizon 2014 Europa (Johnson y al., 2014) apunta a un nivel insuficiente de competencia digital en niños y adolescentes europeos. Sin embargo, se perciben así mismos como expertos y competentes en TIC (Fernández y Fernández, 2016), atribuyendo expectativas muy elevadas hacia la tecnología, donde el aprendizaje suele ser independiente o autodidacta. Hemos de tener en cuenta que los jóvenes universitarios dedican un tiempo elevado al uso del teléfono móvil y de Internet (Garrote et al., 2018). Es desde esta perspectiva donde la digitalización en las diferentes áreas del conocimiento modifica y determina su dominio y nuestra interacción con los contenidos, tanto para afrontar las necesidades de un puesto de trabajo, como para el mero hecho de generar un nuevo saber (Fombona y Pascual, 2013). Y es aquí donde el docente ha de guiar al alumnado en la construcción de su aprendizaje dentro de la llamada sociedad del conocimiento (Rangel, 2015).

Diferentes investigaciones han estudiado la competencia digital, su evaluación en diferentes contextos y niveles (Fernández y Torres, 2015) y a través de diferentes metodologías, siendo la autoevaluación una estrategia útil para los docentes en prácticas (Maderick et al., 2015). Por otra parte, los estudios se han centrado en la interdisciplinariedad y la influencia entre diferentes factores y la competencia digital (González et al., 2015). Como señalan Pérez, et al. (2016), el estudio de la competencia digital en el contexto educativo ha sido abordado en la Educación Secundaria pero sobre todo en el contexto universitario (Sendín et al., 2014), en el que la competencia digital, su evaluación y desarrollo ha sido objeto de varias investigaciones que identifican ciertas prácticas TIC habitualmente realizadas por una nueva generación Net o generación en red (Valtonen, 2011). Cózar-Gutiérrez (2016) destacan que los estudiantes tienen un conocimiento adecuado de las herramientas TIC y que fundamentalmente las emplean para la comunicación social y el ocio y sin embargo no son capaces de transferir ese conocimiento a las habilidades para la enseñanza y el aprendizaje, ni tampoco al proceso de construcción de conocimiento sistematizado (Gabarda et al., 2017; Castellanos et al., 2017; Cabezas et al., 2017). Resultados similares se habían obtenido en estudios previos (Prendes et al., 
2010) con la misma tipología de alumnado (estudiantes de Magisterio) indicando que si bien hacen uso de la tecnología no son capaces de optimizar su rendimiento en el trabajo con TIC. Es necesario realizar un seguimiento de esta evolución y analizar qué nivel de competencia digital tienen los estudiantes cuando llegan a la universidad y poder garantizar, a través de su formación, que esa competencia ha sido adquirida al finalizar sus estudios de grado para su incorporación al mercado profesional como nuevos profesores.

De forma generalizada en los planes de estudio de las diferentes titulaciones no se tiene en cuenta la competencia digital necesaria para abordar una titulación desde el enfoque más tecnológico, lo cual repercute en el diseño de las actividades y su organización (Prendes et al., 2018). De ahí que consideremos necesario partir del conocimiento autopercibido en competencia digital de los estudiantes del primer curso de la titulación de magisterio antes de que inicien su formación específica en este campo como futuros docentes. Hemos de tener en cuenta que la formación competencial del futuro profesorado respecto a las TIC, ha de partir de una competencia digital media o avanzada que ha sido adquirida en la etapa previa de formación. Por lo tanto, el objetivo general de la presente investigación es realizar un diagnóstico de las necesidades formativas de los estudiantes de primer curso del título de Grado de Maestro en Educación Primaria, enfatizando nivel de dominio en la competencia digital como competencia transversal. Entendemos por competencia "la capacitación de movilizar los recursos para poder hacer, pero no entendida como mera aplicación, ni como una reconstrucción, sino como un saber hacer con valor añadido, el de la singularidad de cada persona" (Pérez-Ferra, 2011, p. 95). En virtud de los resultados se pretende ajustar la planificación formativa mediante el diseño de actuaciones que contribuyan a la mejora de esta competencia. Para lograr este objetivo se establecen los siguientes objetivos específicos: 1) Conocer los niveles de competencia digital por áreas competenciales: Información, Comunicación, Creación de Contenidos, Seguridad, y Resolución de Problemas; 2) Conocer qué posibles implicaciones tienen los resultados que se obtienen, en términos de formación y de aprendizaje del alumnado y estudiar la posibilidad de una inclusión curricular a partir de los resultados.

\section{METODOLOGÍA}

El presente estudio es una investigación descriptivo-exploratoria ex post-facto, que busca definir propiedades y describir características y perfiles de personas, comunidades, grupos o cualquier otro fenómeno a través del análisis, relacionando una serie de cuestiones y midiendo la información obtenida sobre cada una de ellas para describir lo investigado (Bisquerra, 2009).

\section{Características de la muestra}

En este estudio, realizado en 2018, participaron un total de 559 estudiantes del primer curso del título de Maestro en Educación Primaria impartido en las Universidades de Granada, Jaén y Oviedo. La muestra se ha establecido siguiendo un método no probabilístico de conveniencia pues se pretendía seleccionar para el análisis, al conjunto de estudiantes. El $11,6 \%$ de los estudiantes encuestados pertenecen a la Universidad de Granada, el $53 \%$ a la Universidad de Jaén y el $35,2 \%$ a la Universidad de Oviedo (Tabla1). Del total de participantes ( $\mathrm{N}=559)$, el $75,8 \%$ son mujeres. Atendiendo a otros datos del alumnado, el $82,8 \%$ ha estudiado el Bachillerato o Formación Profesional en centros públicos y el $64,6 \%$ afirma que lo ha hecho en un centro que tenía implantado el Programa de TIC. El 90\% de los estudiantes utiliza el Smartphone/iphone como recurso tecnológico habitual. Por otro lado, en relación a la formación recibida con anterioridad en el uso de las TIC el $64,6 \%$ del alumnado ha adquirido la destreza tecnológica a través de auto-aprendizaje y un $20 \%$ dice haber adquirido estas destrezas en el centro en el que realizó sus estudios de bachillerato. Estos datos coinciden con lo señalado por Pérez, et al. (2016) en otras etapas educativas. Es de reseñar también que un $45 \%$ dice haber recibido formación en TIC y que esta ha sido presencial para el $62,6 \%$. El acceso a internet, tampoco ofrece problemas ya que el $99,3 \%$ tiene acceso a internet desde su domicilio.

Tabla 1: Características de la muestra

\begin{tabular}{|l|l|r|c|}
\hline \multirow{2}{*}{ Universidad } & Univ. Granada & $11,6 \%$ & $\mathrm{n}=64$ \\
\cline { 2 - 4 } & Univ. Jaén & $53 \%$ & $\mathrm{n}=296$ \\
\cline { 2 - 4 } & Univ. Oviedo & $35,2 \%$ & $\mathrm{n}=197$ \\
\hline \multirow{2}{*}{ Sexo } & Mujeres & $75,8 \%$ & $\mathrm{n}=436$ \\
\cline { 2 - 4 } & Hombres & $24,2 \%$ & $\mathrm{n}=135$ \\
\hline Educación Secundaria con Programa TIC & $\mathrm{Si}$ & $64,6 \%$ & $\mathrm{n}=361$ \\
\hline \multirow{2}{*}{ Formación previa en TIC } & $\mathrm{Si}$ & $45 \%$ & $\mathrm{n}=252$ \\
\cline { 2 - 4 } & Autoaprendizaje & $64,6 \%$ & $\mathrm{n}=361$ \\
\hline \multirow{2}{*}{ Formación previa específica } & Presencial & $62,1 \%$ & $\mathrm{n}=347$ \\
\cline { 2 - 4 } & Virtual & $37 \%$ & $\mathrm{n}=207$ \\
\hline
\end{tabular}




\section{Instrumento}

Para el desarrollo de la recogida de la información, se determinó que el método más adecuado era la encuesta, y para ello se diseñó un instrumento ad hoc denominado "Cuestionario de Percepciones sobre la competencia digital" (C-CompDigital) teniendo en cuenta las fases implicadas en el desarrollo de escalas de medida (Spector, 1992), la selección empírica de ítems (Prat y Doval, 2003) así como una adecuada distribución y claridad informativa. Se han respetado las áreas de competencia digital en las cuales existe mayor consenso y que han sido definidas por el INTEF (2017) en el Marco Común Europeo de Competencia Digital Docente (MCECD). Las áreas de competencia digital descritas en el documento son: Información, Comunicación, Creación de Contenidos, Seguridad, Resolución de Problemas. Hemos de tener en cuenta las limitaciones que puede tener un instrumento de diagnóstico basado en la percepción del propio grado de adquisición de la competencia por cada sujeto, pero consideramos que puede ser una guía orientativa que nos ayude a definir un programa formativo mejor orientado. Se usó una escala aditiva tipo Likert de 5 ítems para conocer el grado de conformidad del encuestado con lo que le proponíamos.

El cuestionario quedó conformado por 5 dimensiones que hacen referencia al uso de las TIC como herramientas de información, de comunicación, para la creación de contenidos, el uso seguro de las TIC y la resolución de problemas. Además, se determinaron 12 variables descriptivas a fin de conocer cómo inciden en el uso de las TIC. Con la intención de asegurar la validez de contenido y la aplicabilidad del instrumento, en su primera versión, fue sometido al juicio de expertos (12 profesores especialistas en TIC, de las tres universidades participantes) quienes evaluaron la pertinencia al objeto de estudio y adecuación a la muestra empleando para ello una escala de 1 a 5 . Se seleccionaron solamente aquellos ítems que habían obtenido una calificación de 3 puntos o superior a ellas. El resultado final fue un cuestionario compuesto por 51 ítems. Posteriormente, se llevó a cabo un estudio piloto realizado sobre una muestra de 32 estudiantes de magisterio del $1^{\circ}$ curso que permitió, reformular la redacción de algunos ítems. Previamente a la determinación de la validez del constructo se aplicó la prueba de esfericidad de Barlett, a fin de probar la hipótesis nula de que la matriz de correlaciones es la matriz identidad; es decir que las variables están correlacionadas con la población, aportando el test un resultado de 0,000 , razón por la que se rechaza la hipótesis nula a un nivel de significación del $5 \%$, que permite realizar el análisis. El cuestionario fue aplicado digitalmente en el aula de informática con la supervisión del investigador. Posteriormente se determinó la fiabilidad de la escala, a fin de definir la consistencia interna. El Alfa de Cronbach respecto a la totalidad de la escala es de $(0,786)$. La eliminación de cada ítem respecto a la totalidad, aporta los siguientes datos (ítem $n^{\circ} 33=0,770<0,789=$ ítem $\left.n^{\circ} 22\right)$. No se suprime ningún ítem, ya que al eliminar cada ítem, el resultado no aporta un alfa superior al de la totalidad de la escala; es decir 0,791. Se aplicó el Alfa de las dos mitades para conocer el nivel de consistencia entre las dos partes del cuestionario; en los ítems impares se obtuvo un alfa $=0,695$; y en los pares 0,710; por consiguiente, hay una consistencia razonable en la fiabilidad de los ítems pares respecto a los impares. La escala final obtenida queda integrada por 51 ítems. Una vez finalizada la recogida de datos se procedió a analizar dicha información a través del programa SPSS 24.

\section{RESULTADOS}

La siguiente categorización en las áreas en las que se ha estructurado la competencia digital facilita el análisis y comprensión de los resultados. Los datos se han calculado en base a los 559 participantes en el estudio, seleccionando para su presentación los porcentajes de respuesta válidos. Los resultados se muestran en función de las dimensiones de las que consta el instrumento. El análisis descriptivo de las 5 áreas competenciales del instrumento, recoge los estadísticos en forma de media y desviación típica. Así, en cuanto al área competencial Acceso a la Información (Tabla 2) en la unidad de competencia Navegación, búsqueda y filtrado de información destaca que el $70.6 \%$ de los estudiantes localiza información digitalizada a través de palabras clave y filtros que limitan la búsqueda $(M=3.32, \sigma=1.2)$, pero solo el $53.6 \%$ es capaz de configurar el navegador de su ordenador $(\mathrm{M}=3.03, \sigma=1.37)$. En la unidad de competencia Evaluación de la información el $88.5 \%$ de los estudiantes son capaces de utilizar los recursos de la red para realizar actividades de clase $(M=3.65, \sigma=1.48)$, sin embargo, solo el $53 \%$ tiene en cuenta las restricciones de los recursos publicados con derechos de autor $(M=3.09 \sigma=1.48)$. En la unidad de competencia Almacenamiento y recuperación de información, el $88 \%$ de los encuestados se muestra competente en el almacenamiento de la información en función de los formatos de los archivos ( $M=3.95, \sigma=1,11)$. $Y$ el $70 \%$ dice saber hacerlo en espacios de almacenamiento externo (nube, disco duro, memorias USB). ( $M=3.72, \sigma=1.32$ ). Sin embargo, el $64.5 \%$ de los estudiantes no utilizan programas informáticos para organizar y etiquetar la información obtenida de internet $(M=2.38, \sigma=1.45)$. Con el fin de presentar un promedio de adquisición de esta subcompetencia establecemos la relación entre la escala Likert y los niveles en los que se suele graduar la adquisición de competencias (MEC, 2013), siendo el rango 1-2 el correspondiente a un nivel bajo, el rango 3 a un nivel medio y el rango 4-5 a un nivel avanzado. Estos niveles nos permiten, finalmente, puntuar y evaluar la competencia digital de los estudiantes, asociando a cada uno de ellos un valor numérico. Teniendo en cuenta esta relación señalamos que los estudiantes tienen un promedio en la subcompetencia Acceso a la Información de 3.3 lo que equivale a un nivel medio. 
Tabla 2: Área competencial: Acceso a la Información

\begin{tabular}{|c|c|c|c|}
\hline $\begin{array}{l}\text { Unidad de } \\
\text { competencia }\end{array}$ & Ítem & $\begin{array}{c}\text { Media } \\
\text { Rango 1-5 }\end{array}$ & $\begin{array}{l}\text { Desviación } \\
\text { estándar }\end{array}$ \\
\hline \multirow{4}{*}{$\begin{array}{l}\text { Navegación, } \\
\text { búsqueda y } \\
\text { filtrado }\end{array}$} & $\begin{array}{l}\text { 1. Configuras el navegador web de tu ordenador cuando lo } \\
\text { consideras necesario. }\end{array}$ & 3.03 & 1.37 \\
\hline & 2. Seleccionas fuentes de información de interés profesional. & 3.15 & 1.02 \\
\hline & $\begin{array}{l}\text { 3. Localizas información digitalizada utilizando palabras clave y } \\
\text { filtros para ajustar y limitar tu búsqueda. }\end{array}$ & 3.32 & 1.28 \\
\hline & 4. Usas distintos buscadores para localizar información y recursos. & 3.14 & 1.34 \\
\hline \multirow[t]{3}{*}{$\begin{array}{l}\text { Evaluación de la } \\
\text { información }\end{array}$} & $\begin{array}{l}\text { 5. Tienes en cuenta las restricciones de los recursos educativos } \\
\text { publicados con derechos de autor. }\end{array}$ & 3.09 & 1.48 \\
\hline & $\begin{array}{l}\text { 6. Utilizas recursos educativos disponibles en Internet en función de } \\
\text { las demandas que te plantean las asignaturas. }\end{array}$ & 3.65 & 1.07 \\
\hline & $\begin{array}{l}\text { 7. Evalúas críticamente la información que localizas en internet } \\
\text { posicionándote sobre su enfoque. }\end{array}$ & 3.36 & 1.08 \\
\hline \multirow{4}{*}{$\begin{array}{l}\text { Almacenamiento } \\
\text { y recuperación } \\
\text { de la } \\
\text { información }\end{array}$} & $\begin{array}{l}\text { 8. Guardas información en diferentes formatos clasificándola de } \\
\text { forma que la puedas recuperar fácilmente. }\end{array}$ & 3.95 & 1.11 \\
\hline & $\begin{array}{l}\text { 9. Realizas copias de seguridad de la información que consideras } \\
\text { relevante }\end{array}$ & 3.54 & 1.32 \\
\hline & 10. Utilizas espacios de almacenamiento externo con ese fin. & 3.72 & 1.32 \\
\hline & $\begin{array}{l}\text { 11. Utilizas algún tipo de programa informático para organizar, } \\
\text { etiquetando los recursos conseguidos en la red internet }\end{array}$ & 2.38 & 1.45 \\
\hline
\end{tabular}

En el área competencial Comunicación (Tabla 3) los datos indican un alto porcentaje de alumnado que tiene adquirida la unidad de competencia de Interacción a través de los medios digitales $(M=4.73 ; \sigma=0.68)$. En la unidad relacionada con la capacidad para Compartir información y contenidos encontramos más dificultades entre los estudiantes, así el 66,4\% utiliza programas y aplicaciones para crear presentaciones digitales para el aula ( $M=3.21 ; \sigma=1.30)$, sin embargo solo el $47 \%$ difunde en línea las presentaciones creadas.

Tabla 3: Área competencial: Comunicación

\begin{tabular}{|c|c|c|c|}
\hline $\begin{array}{l}\text { Unidad de } \\
\text { competencia }\end{array}$ & Ítem & Media & $\begin{array}{l}\text { Desviació } \\
n \\
\text { estándar }\end{array}$ \\
\hline \multirow{2}{*}{$\begin{array}{l}\text { Interacción a } \\
\text { través de los } \\
\text { medios digitales }\end{array}$} & $\begin{array}{l}\text { 12. Utilizas al menos tres herramientas digitales distintas para } \\
\text { interactuar con los demás. }\end{array}$ & 4.73 & .68 \\
\hline & $\begin{array}{l}\text { 13. Cuando navegas, interaccionas con diferentes fuentes de } \\
\text { información digital. }\end{array}$ & 4.23 & .90 \\
\hline \multirow{4}{*}{$\begin{array}{l}\text { Compartir } \\
\text { información y } \\
\text { contenidos }\end{array}$} & $\begin{array}{l}\text { 14. Cuando compartes información o contenidos obtenidos en línea, } \\
\text { citas al autor y a la fuente, siguiendo las normas existentes. }\end{array}$ & 3.13 & 1.25 \\
\hline & $\begin{array}{l}\text { 15. Seleccionas para compartir en red, noticias y recursos localizados } \\
\text { en diversas páginas web, foros y otras comunidades en línea. }\end{array}$ & 3.06 & 1.15 \\
\hline & $\begin{array}{l}\text { 16. Utilizas programas y aplicaciones para crear presentaciones } \\
\text { digitales proyectables. }\end{array}$ & 3.21 & 1.30 \\
\hline & $\begin{array}{l}\text { 17. Difundes las presentaciones digitales creadas a través de envío } \\
\text { por correo, redes sociales, subida a repositorios, etc. }\end{array}$ & 2.74 & 1.31 \\
\hline \multirow{2}{*}{$\begin{array}{l}\text { Participación } \\
\text { ciudadana en } \\
\text { línea }\end{array}$} & $\begin{array}{l}\text { 18. Participas activamente en al menos dos comunidades virtuales y } \\
\text { redes sociales. }\end{array}$ & 4.47 & 1.03 \\
\hline & $\begin{array}{l}\text { 19. Aprovechas las posibilidades que te ofrecen los medios digitales } \\
\text { incluyendo a las redes sociales para promover la participación social } \\
\text { de la ciudadanía. }\end{array}$ & 3.29 & 1.19 \\
\hline $\begin{array}{l}\text { Colaboración con } \\
\text { medios digitales }\end{array}$ & $\begin{array}{l}\text { 20. Usas herramientas de trabajo colaborativo en línea para crear } \\
\text { conocimiento compartido. }\end{array}$ & 2.94 & 1.08 \\
\hline \multirow[t]{2}{*}{ Netiqueta } & $\begin{array}{l}\text { 21. Consultas las disposiciones legales que regulan los derechos y } \\
\text { deberes de quienes emitimos y recibimos mensajes digitales. }\end{array}$ & 2.40 & 1.38 \\
\hline & $\begin{array}{l}\text { 22. Cuando emites y recibes mensajes digitales cumples las normas } \\
\text { legales que regulan tal transferencia de información. }\end{array}$ & 3.72 & 1.97 \\
\hline
\end{tabular}


Tabla 3 (Continuación)

\begin{tabular}{|l|l|c|c|}
\hline Netiqueta & $\begin{array}{l}\text { 23. Tienes presente las características socioculturales de las } \\
\text { audiencias hacia los que diriges tus mensajes digitales que creas para } \\
\text { difundirlos a través de internet. }\end{array}$ & 3.47 & 1.45 \\
\hline $\begin{array}{l}\text { Gestión de la } \\
\text { identidad digital }\end{array}$ & $\begin{array}{l}\text { 24. Gestionas tu identidad digital, cuidando de hacer públicos solo } \\
\text { aquellos datos personales que deseas que se conozcan. }\end{array}$ & 4.41 & .95 \\
\cline { 2 - 5 } & 25. Rastreas tu huella digital en Internet para conocer su difusión. & 2.86 & 1.90 \\
\hline
\end{tabular}

En relación a la Participación ciudadana en línea, la mayoría $(M=4.47 ; \sigma=1.3)$ participa activamente al menos en dos comunidades virtuales. Sin embargo solo el $67.5 \%$ suelen utilizar herramientas de trabajo colaborativo para crear conocimiento compartido $(\mathrm{M}=2.9 ; \sigma=1.08)$. En relación a la unidad de competencia Netetiqueta, o reglas de etiqueta en la red, el $65.5 \%(M=2.4 ; \sigma=1.38)$ no consulta las disposiciones legales sobre derechos y deberes en la emisión y recepción de mensajes, y solo el $58 \%(M=3.7 ; \sigma=1.97)$ tiene en cuenta esas normas. Era importante conocer como gestionan en esa comunicación su identidad digital y observamos que el $94 \%$ $(M=4.41 ; \sigma=0.95)$ dice gestionarla sin aportar datos personales que no desea hacer públicos. Pero, solo el $47 \%$ rastrea su huella digital en Internet para conocer su difusión. El promedio de adquisición de la subcompetencia Comunicación es de 3.4 que equivale a un nivel medio.

En el área competencial Creación de Contenidos, los datos indican en general valores por debajo de 3 (Tabla 4). En la unidad de competencia Desarrollo de contenidos observamos que el $87.5 \%$ comparte materiales sencillos en la red $(M=3.76 ; \sigma=1.06)$, el $79.5 \%$ diseña y crea presentaciones digitales $(M=3.4 ; \sigma=1.14)$, sin embargo solo el $43.6 \%$ gestiona espacios web donde publicar contenidos multimedia ( $M=2.5 ; \sigma=1.31)$, el $34 \%$ crea audios digitales mezclando voz y música $(\mathrm{M}=2.33$; $\sigma=1.23)$ y el $53 \%$ crea videos propios insertando diferentes recursos $(M=2.81 ; \sigma=1.25)$. En la unidad Integración y estructuración el $55.6 \%$ afirman respetar las normativas que regulan las licencias de uso de materiales creados $(M=3.37 ; \sigma=1.54)$, pero solo el $35 \%$ se preocupa por conocerlas $(\mathrm{M}=2.5 ; \sigma=1.35)$ y el $29 \%$ dice preocuparse por los procedimientos para liberalizar sus derechos para hacer su obra de dominio público y gratuito $(\mathrm{M}=2.64 ; \sigma=1.58)$. En relación a la unidad Programación encontramos un $69 \%$ capaz de modificar la configuración básica de las herramientas y medios digitales en función de sus necesidades $(\mathrm{M}=3.31 ; \sigma=1.30)$. El promedio de adquisición de la subcompetencia Creación de Contenidos es de 3.3 lo cual equivale a un nivel medio.

Tabla 4: Área competencial: Creación de Contenidos

\begin{tabular}{|c|c|c|c|}
\hline $\begin{array}{l}\text { Unidad de } \\
\text { competencia }\end{array}$ & Ítem & Media & $\begin{array}{l}\text { Desviación } \\
\text { estándar }\end{array}$ \\
\hline \multirow{6}{*}{$\begin{array}{l}\text { Desarrollo de } \\
\text { contenidos }\end{array}$} & 26. Compartes materiales digitales sencillos en la red. & 3.76 & 1.06 \\
\hline & $\begin{array}{l}\text { 27. Gestionas espacios web donde publicar contenidos multimedia } \\
\text { elaborados por ti. }\end{array}$ & 2.52 & 1.31 \\
\hline & $\begin{array}{l}\text { 28. Creas mensajes adaptados en forma y contenido a las características } \\
\text { de los canales de comunicación digital en los que vas a difundir. }\end{array}$ & 3.58 & 1.23 \\
\hline & 29. Creas audios digitales mezclando voz y música. & 2.33 & 1.23 \\
\hline & $\begin{array}{l}\text { 30. Diseñas y creas presentaciones digitales combinando imágenes, } \\
\text { gráficos y textos. }\end{array}$ & 3.41 & 1.14 \\
\hline & $\begin{array}{l}\text { 31. Creas vídeos propios insertando fotografías, gráficos, fragmentos de } \\
\text { películas y textos. }\end{array}$ & 2.81 & 1.25 \\
\hline \multirow[t]{4}{*}{$\begin{array}{l}\text { Integración y } \\
\text { estructuración }\end{array}$} & $\begin{array}{l}\text { 32. Usas programas de edición digital (de audio y/o vídeo) para modificar } \\
\text { contenidos creados por ti o por otros autores. }\end{array}$ & 2.75 & 1.81 \\
\hline & $\begin{array}{l}\text { 33. Te preocupas de conocer las normativas que regulan los derechos de } \\
\text { autor según el tipo de licencia elegida para protegerlos. }\end{array}$ & 2.51 & 1.35 \\
\hline & $\begin{array}{l}\text { 34. Respetas las obligaciones y derechos previstos en las normativas que } \\
\text { regulan las licencias de uso de los materiales creados por los autores. }\end{array}$ & 3.37 & 1.54 \\
\hline & $\begin{array}{l}\text { 35. Te preocupas por conocer los procedimientos por los que un autor } \\
\text { liberaliza sus derechos y hace su obra de dominio público y gratuito. }\end{array}$ & 2.64 & 1.58 \\
\hline Programación & $\begin{array}{l}\text { 36. Modificas la configuración básica de las herramientas y medios digitales } \\
\text { de los que dispones, para adaptarla a tus necesidades y limitaciones. }\end{array}$ & 3,31 & 1,30 \\
\hline
\end{tabular}

En el área competencial Seguridad se observa (Tabla 5) que en general los alumnos están concienciados con la seguridad de sus herramientas digitales y la practican, así en relación a la protección de los dispositivos, el $88.2 \%$ realiza el mantenimiento y protección de sus dispositivos $(M=3.88 ; \sigma=1.11)$ y el $70 \%$ sabe cómo 
proceder cuando detecta una amenaza de seguridad en sus equipos $(M=3.41 ; \sigma=1.25)$. En relación a la protección de sus datos, también se muestra cauteloso y más del $90 \%$ protege sus datos personales y su identidad digital $(\mathrm{M}=4.3 ; \sigma=1.02)$. La protección de la salud también es contemplada por los estudiantes y el $92.3 \%$ se preocupa por los riesgos asociados con un uso inadecuado de los dispositivos electrónicos $(M=3.66$; $\sigma=1.29$ ). La protección del entorno también es tenida en cuenta por los estudiantes de magisterio y así el $66.4 \%$ hace un uso óptimo de los dispositivos digitales para que tengan un menor impacto en el medio ambiente $(M=3.38 ; \sigma=1.3)$ y el $80 \%$ adopta medidas eficaces de ahorro energético a fin de alargar la durabilidad de las baterías de los dispositivos $(\mathrm{M}=3.7 ; \sigma=1.76)$. El promedio de adquisición de la subcompetencia Seguridad de 3.8 lo cual equivale a un nivel medio alto.

Tabla 5: Área competencial: Seguridad

\begin{tabular}{|l|l|c|c|}
\hline \multicolumn{1}{|c|}{$\begin{array}{c}\text { Unidad de } \\
\text { competencia }\end{array}$} & \multicolumn{1}{|c|}{ Ítem } & $\begin{array}{c}\text { Media } \\
\text { Desviación } \\
\text { estándar }\end{array}$ \\
\hline $\begin{array}{l}\text { Protección de } \\
\text { dispositivos }\end{array}$ & $\begin{array}{l}\text { 37. Realizas operaciones básicas de mantenimiento y protección de } \\
\text { los dispositivos que utilizas: actualizaciones de sistemas, antivirus y } \\
\text { contraseñas de acceso, etc. }\end{array}$ & 3.88 & 1.11 \\
\cline { 2 - 5 } & $\begin{array}{l}\text { 38. Sabes cómo actuar cuando detectas una amenaza de seguridad } \\
\text { en tus dispositivos y equipos. }\end{array}$ & 3.41 & 1.25 \\
\hline $\begin{array}{l}\text { Protección de datos } \\
\text { personales e } \\
\text { identidad digital }\end{array}$ & 39. Proteges los datos personales sensibles. & 4.32 & 1.02 \\
\cline { 2 - 5 } $\begin{array}{l}\text { Protección de la } \\
\text { salud }\end{array}$ & $\begin{array}{l}\text { 41. Proteges tu identidad digital y la de los demás. } \\
\text { uso inadecuado de dispositivos y equipos electrónicos. }\end{array}$ & 4.26 & 3.66 \\
\hline $\begin{array}{l}\text { Protección del } \\
\text { entorno }\end{array}$ & $\begin{array}{l}\text { 42. Haces un uso óptimo de los dispositivos digitales para que tengan } \\
\text { el menor impacto sobre el medio ambiente. }\end{array}$ & 3.38 & 1.29 \\
\cline { 2 - 5 } & $\begin{array}{l}\text { 43. Adoptas medidas eficaces de ahorro energético y de } \\
\text { alargamiento de la duración de las baterías de tus dispositivos } \\
\text { digitales. }\end{array}$ & 3.70 & 1.30 \\
\hline
\end{tabular}

En el área competencial Resolución de Problemas (Tabla 6) se han analizado 4 aspectos y es la unidad relacionada con la resolución de cuestiones técnicos donde más estudiantes dicen no ser competentes; solo el $61 \%$ de los encuestados dice identificar y resolver problemas técnicos no complejos $(M=2.96 ; \sigma=1.19)$. El $78 \%$ utiliza los foros especializados para resolver problemas o dudas $(M=3.37 ; \sigma=1.13)$ y el $93 \%$ acude a las tecnologías para buscar soluciones innovadoras que faciliten su aprendizaje $(M=3.91 ; \sigma=0.97$. Pero en general casi el $80 \%$ reconoce tener carencias en el uso de los medios digitales con fines de aprendizaje profesional $(M=3.7 ; \sigma=1.17)$, pero no se retraen a la hora de experimentar con la tecnología para cubrir sus lagunas en la competencia digital $(M=3.38 ; \sigma=1.14)$. El promedio de adquisición de la subcompetencia Resolución de Problemas es de 3,3 lo cual equivale a un nivel medio.

Tabla 6: Área competencial: Resolución de Problemas

\begin{tabular}{|c|c|c|c|}
\hline $\begin{array}{l}\text { Unidad de } \\
\text { competencia }\end{array}$ & Ítem & Media & $\begin{array}{l}\text { Desviación } \\
\text { estándar }\end{array}$ \\
\hline \multirow{2}{*}{$\begin{array}{l}\text { Resolución de } \\
\text { problemas } \\
\text { técnicos }\end{array}$} & $\begin{array}{l}\text { 44. Identificas un problema técnico explicando con claridad en qué } \\
\text { consiste el mal funcionamiento. }\end{array}$ & 2.92 & 1.26 \\
\hline & $\begin{array}{l}\text { 45. Resuelves problemas técnicos no complejos relacionados con } \\
\text { dispositivos y entornos digitales habituales con la ayuda de un manual } \\
\text { o información técnica disponible. }\end{array}$ & 2.96 & 1.19 \\
\hline \multirow{2}{*}{$\begin{array}{l}\text { Identificación de } \\
\text { necesidades y } \\
\text { respuestas } \\
\text { tecnológicas }\end{array}$} & $\begin{array}{l}\text { 46. Utilizas entornos virtuales para seguir cursos en red para tu } \\
\text { formación. }\end{array}$ & 2.70 & 1.25 \\
\hline & $\begin{array}{l}\text { 47. Consultas foros especializados que te ayuden a resolver dudas } 0 \\
\text { problemas }\end{array}$ & 3.37 & 1.13 \\
\hline \multirow{2}{*}{$\begin{array}{l}\text { Innovación y uso } \\
\text { de las tecnologías } \\
\text { de forma creativa }\end{array}$} & $\begin{array}{l}\text { 48. Usas las tecnologías en tu actividad académica para buscar } \\
\text { soluciones alternativas e innovadoras que faciliten el aprendizaje. }\end{array}$ & 3.91 & .97 \\
\hline & $\begin{array}{l}\text { 49. Utilizas las tecnologías en tu labor diaria para gestionar soluciones } \\
\text { innovadoras y participar en proyectos creativos. }\end{array}$ & 3.53 & 1.12 \\
\hline \multirow{2}{*}{$\begin{array}{l}\text { Identificación de } \\
\text { lagunas en la } \\
\text { competencia } \\
\text { digital }\end{array}$} & $\begin{array}{l}\text { 50. Reconoces las carencias en el uso de medios digitales con fines de } \\
\text { aprendizaje profesional docente. }\end{array}$ & 3.70 & 1.17 \\
\hline & $\begin{array}{l}\text { 51. Experimentas con nuevas tecnologías digitales que te ayudan a } \\
\text { cubrir posibles lagunas en la competencia digital, necesaria para tu } \\
\text { aprendizaje. }\end{array}$ & 3.38 & 1.14 \\
\hline
\end{tabular}


En resumen, los promedios en cada una de las dimensiones de la competencia digital oscilan entre 3,3 y 3,8 que en la asociación establecida entre rangos y niveles equivaldría a un nivel medio de la competencia digital (Tabla 7). El área donde los estudiantes tienen una media mayor (3,8 sobre 5) cercano a un nivel avanzado es la de seguridad en la protección de dispositivos, detección de amenazas de seguridad, protección de datos personales e identidad personal, y uso responsable de los dispositivos.

Tabla 7: Promedios de la competencia digital

\begin{tabular}{|l|r|}
\hline \multicolumn{1}{|c|}{ Competencia digital } & Promedio \\
\hline 1. Acceso a la Información & 3.3 \\
\hline 2. Comunicación & 3.4 \\
\hline 3. Creación de Contenidos & 3.3 \\
\hline 4. Seguridad & 3.8 \\
\hline 5. Resolución de Problemas & 3.3 \\
\hline
\end{tabular}

\section{DISCUSIÓN}

Los aspectos detectados en este proyecto y su discusión, han generado el diseño de un programa formativo al efecto, basado en un aprendizaje práctico y siguiendo las recomendaciones de la perspectiva socioconstructivista (Ortega, 2013). En primer lugar es importante analizar las múltiples y complejas dimensiones que aparecen cuando abordamos la competencia digital, así esta investigación viene a completar otros estudios previos, como los realizados por Strijbos et al., (2015), Cózar-Gutiérez et al. (2016), y por Prendes et al. (2010) que analizan de una forma sectorial algunas de las opciones de las TIC. Coincidimos en resaltar que el desarrollo de estas competencias está rodeado de un componente motivador, quizás por su carácter eminentemente práctico (Cózar-Gutiérez et al., 2016). Los resultados de las dimensiones Información, Comunicación y Creación de Contenidos son cada vez más importantes y reiteran carencias en el conocimiento sobre la normativa y el uso de autorías responsables, el conocimiento y respeto por las licencias que permite cada documento, todo ello para hacer una producción rigurosa de materiales. Se observa que la competencia digital se relaciona especialmente con aspectos sobre la gestión de elevadas cantidades de información, y en este sentido la investigación muestra un desconocimiento sobre cómo manejar tantos datos. Esta circunstancia parece ser más importante con el paso del tiempo y el incremento del caudal informático. Los datos a nivel global coinciden con los hallazgos de Espuny et al., (2010) en tanto que los estudiantes no dominan los programas especializados en reordenar y clasificar la información. Resultados similares parecen reiterarse en otros estudios realizados a nivel universitario, en cuanto al desconocimiento en el manejo de estas herramientas (Gabarda et al., 2017; Castellanos et al., 2017). Esto nos sugiere la necesidad de incluir esta formación al inicio de la carrera.

Resulta especialmente novedosa la posibilidad que ofrecen los recursos digitales para la Creación de Contenidos y el acceso a materiales elaborados por otros autores obliga a conocer las licencias específicas de uso. Cabezas et al. (2017) también detectan bajos resultados en el conocimiento del manejo de herramientas para la edición de imagen y sonido. Por ello parece importante una formación al efecto, y coincidimos en esta prioridad con los estudios de Castellanos et al. (2017) sobre estos niveles de capacitación, sobre todo para la interacción de contenidos en las redes sociales, herramientas de comunicación y de elaboración de documentos. Al contrario, la competencia relacionada con la Seguridad es buena y parece que estos estudiantes son usuarios respetuosos y seguros. Estos datos coinciden con estudios en otros contextos en los que se afirma que los jóvenes adoptan restricciones de acceso en sus perfiles de las redes sociales (Madden et al., 2013).

La última dimensión analiza la Resolución de Problemas informáticos, en este ámbito se detectan algunas carencias formativas importantes, a este respecto autores como Prendes et al. (2010) destacan la importancia de las competencias técnicas que deben poseer los futuros maestros. Así mismo, los datos de la investigación coinciden con los resultados de Cabero y Llorente (2006) al describir a los estudiantes como moderadamente competentes en la búsqueda de soluciones a los problemas que se plantean y cómo acceden a fuentes de información especializadas y en red para solucionar sus cuestiones en la gestión técnica. Consideramos al igual que estos autores, la necesidad de incluir estos conocimientos específicos como base para abordar los cambios tecnológicos y los nuevos problemas que surgen en el aula que utiliza habitualmente los recursos digitales.

Al cuantificar estas variables en la competencia digital de los estudiantes de primer curso en el grado de Educación de Primaria se revela la situación real derivada de su formación previa en la enseñanza secundaria y puede ser un buen momento para replantear la propia programación educativa del docente en materia TIC. 
Este estudio también permite conocer el repertorio de dimensiones de la competencia digital sobre las que hay que formar a los estudiantes y sobre qué aspectos se debe profundizar. Consideramos que el dinamismo tecnológico, y los cambios que suceden y generan las TIC, han de impregnar y renovar las diferentes formas de enseñanza. Es una necesidad de innovación constante en el proceso de formación inicial del profesorado para que pueda dar respuesta eficaz a los nuevos retos de nuestra sociedad. Dado el carácter exploratorio de este estudio se aconseja una interpretación prudente de los resultados, fundamentalmente a la hora de generalizar los hallazgos a otros contextos. También se ha de tener en cuenta que la distribución de la muestra por universidades no ha sido homogénea y que se ha empleado una herramienta cuantitativa de autopercepción que se podría complementar con una prueba objetiva de medición de la competencia digital.

\section{CONCLUSIONES}

El análisis de la competencia digital en los estudiantes posibilita mostrar sus fortalezas y carencias. Esto es:

1) En el área competencial de Información, desconocen las opciones del software de navegación web, el respeto a los derechos de autor y las restricciones sobre estos documentos. También hay carencias importantes sobre cómo gestionar la elevada cantidad de información a la que habitualmente se accede.

2) En el área competencial de Comunicación, destaca el manejo de múltiples herramientas y comunidades interactivas. También surge la necesidad de mejorar la difusión de información específica. Parece necesario fortalecer el dominio de herramientas de trabajo colaborativo, y controlar y rastrear la huella digital en Internet con pautas para identificar los datos propios, y las reglas de autoría en la producción de mensajes.

3) En el área de Creación de Contenidos se ha evidenciado la necesidad de mejorar las habilidades para elaborar portales web propios donde publicar contenidos. También, deben saber crear audiovisuales eficazmente, con distintos tipos de recursos de carácter educativo.

4) En la competencia de Seguridad, en general los estudiantes se manifiestan competentes, protegen sus dispositivos y saben cómo actuar ante una amenaza de seguridad digital.

5) En el área de Resolución de Problemas, afirman tener carencias en la identificación y resolución de cuestiones técnicas ante la constante evolución tecnológica.

\section{AGRADECIMIENTOS}

Es una investigación dentro del Proyecto I+D+i "Evaluación y desarrollo de dos competencias genéricas en estudiantes de primer año del grado de maestro en educación primaria", ref.: EDU2015-70491-R, en el programa estatal de investigación, desarrollo e innovación orientada a los retos de la sociedad en el marco del plan estatal de investigación científica y técnica de la innovación.

\section{REFERENCIAS}

Baartman, L.K., T.J. Bastiaens, P.A. Kirschner y C. Van Der Vleuten, Evaluating Assessment Quality in Competence-based Education: A qualitative Comparison of two Frameworks, doi: 10.1016/j.edurev.2007.06.001, Educational Research Review, 2(2), 114-129 (2007)

Bisquerra, R. (Coord.), Metodología de investigación educativa, $1^{\text {a }}$ Ed., Madrid, La Muralla (2009)

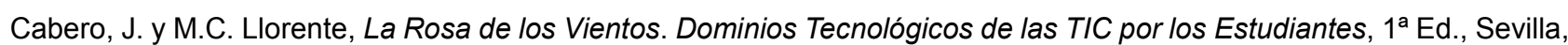
España, Grupo de Investigación Didáctica (2006)

Cabezas, M., S. Casillas, M. Sanches-Ferreira y F.L. Teixeira, ¿Condicionan el Género y la Edad el Nivel de competencia Digital? Un estudio con Estudiantes Universitarios, doi: 10.14201/fjc201715109125, Fonseca, Journal of Comunication, 15, 109-125 (2017)

Castellanos, A., C. Sánchez y F. Calderero, Nuevos Modelos Tecnopedagógicos. Competencia Digital de los Alumnos Universitarios, doi: 10.24320/redie.2017.19.1.1148, Revista Electrónica de Investigación Educativa, 19(1), 1-9 (2017)

Comisión Europea, Competencias Clave para el Aprendizaje Permanente. Un Marco de Referencia Europeo, $1^{\mathrm{a}}$ Ed., Luxemburgo, Oficina de Publicaciones de la Comunidad Europea (2007)

Comisión Europea, Education and Training Monitor, $1^{\mathrm{a}}$ Ed., Bruselas, Oficina Publicaciones Comunidad Europea (2013)

Cózar-Gutiérrez, R., M. De Moya-Martínez, J. Hernández-Bravo y J. Hernández-Bravo, Conocimiento y Uso de las Tecnologías de la Información y la Comunicación (TIC) según el Estilo de Aprendizaje de los Futuros Maestros, doi: 10.4067/S0718-50062016000600010, Formación Universitaria, 9(6), (2016)

Espuny, C., J. González y M. Gisbert, ¿Cuál es la Competencia Digital del Alumnado al llegar a la Universidad? ISSN: 
0212-5374, 28(2), Enseñanza \& Teaching, 113-137 (2010)

Fernández, F.J. y M.J. Fernández, Generation Z’s Teachers and their Digital Skills, doi: 10.3916/C46-2016-10, Comunicar, 46, 97-105 (2016)

Fernández, J.M. y J.A. Torres, Actitudes Docentes y Buenas Prácticas con TIC del Profesorado de Educación Permanente de Adultos en Andalucía, doi: 10.5209/rev_RCED.2015.v26.43812, Revista Complutense de Educación, 26, 33-49 (2015)

Ferrari, A., Digital Competence in Practice: an Analysis of Frameworks, doi: 10.2791/82116, JRC Technical Reports, Comisión Europea (2012)

Fombona, J. y M.A. Pascual, Audiovisual Resources in Formal and Informal Learning: Spanish and Mexican students' Attitudes. International Education Studies, 6(2), 1-11 (2013)

Garrrote-Rojas, D., S. Jiménez-Fernández e I. M. Gómez-Barreto, Problemas Derivados del Uso de internet y el Teléfono Móvil en Estudiantes Universitarios. doi: 104067/S018-50062018000200099, Revista Formación Universitaria, 12 (2), 99 108 (2018)

González, M.P., E.A. Galvis y J.M. Sánchez, Identificación de Factores que Afectan el Desarrollo de la Inclusión Digital, ISNN: 0124-5821, Revista Virtual de La Universidad Católica del Norte, 44, 175-191 (2015)

INTEF, Marco Común de Competencia Digital Docente, $1^{a}$ Ed., Madrid, Instituto Nacional de Tecnologías Educativas y Formación del Profesorado (2017)

Johnson, L., S. Adams-Becker y otros cinco autores, Horizon Report Europe: 2014 Schools Edition, doi: 10.2791/83704, Luxemburgo, Oficina de Publicaciones de la Unión Europea y New Media Consortium (2014)

Madden, M., A. Lenhart y otros cinco autores, Teens, Social Media, and Privacy. Pew Research Center. Washington (2013)

Maderick, J.A., Z. Shaoan, K. Hartley y G. Marchand, Preservice Teachers and Self-assessing Digital Competence, doi: 10.1177\%2F0735633115620432, Journal of Educational Computing Research, 0(0), 1-26 (2015)

Ortega, J., Affective Elearning, una utopía que comienza a hacerse Realidad, ISSN:1695-324X13, Etic@net, 1-4 (2013)

Pérez-Ferra, M. y R. Quijano, Capacidades Docentes del Profesor Universitario para la Gestión del Currículum. En A. Pantoja, M. Zwvierewicz y R. Moraes (Eds.). Diversidad y Adversidad en Educación, 1ª Ed., 73-98, Jaén, Joxman (2011)

Pérez-Escoda, A., A. Castro y M. Fandos, Digital Skills in the Z Generation: Key Questions for a Curricular Introduction in Primary School, doi: 10.3916/C49-2016-07, Comunicar, 49, 71-80 (2016)

Prat, R. y E. Doval, Construcción y Análisis de Escalas, en Lévy, J. y Varela, J. (eds.), Análisis Multivariable para las Ciencias Sociales, $1^{\text {a }}$ Ed., Madrid, Pearson-Prentice Hall (2003)

Prendes, M.P., L. Castañeda e I. Gutiérrez, ICT Competences of Future Teachers, doi: 10.3916/C35-2010-03-11, Comunicar, 35, 175-182 (2010)

Prendes, M.P., I. Gutiérrez y F. Martínez, Competencia digital: una necesidad del profesorado universitario en el siglo XXI, doi: 10.6018/red/56/7, Revista de Educación a Distancia, 56, 1-22 (2018)

Rangel, A., Competencias Docentes Digitales: Propuesta de un Perfil, ISSN: 1133-8482, Píxel-Bit. Revista de Medios y Educación, 46, 235-248 (2015)

Sendín, J., P. Gaona y A. García, Nuevos Medios: Usos Comunicativos de los Adolescentes. Perspectivas desde los Nativos Digitales, ISSN: 1134-1629, Estudios sobre el Mensaje Periodístico, 20(1), 265-280 (2014)

Spector, P.E. Summated rating scale construction: An introduction, doi: 10.4135/9781412986038, Sage University, Paper Series on Quantitative Applications in the Social Sciences (1992)

Strijbos, J., N. Engels y K. Struyven, Criteria and Standars of Generic Competences at Bachelor Degree Level: a Review Study, doi: 10.1016/j.edurev.2015.01.001, Educational Research Review, 13, 18-32 (2015)

Valtonen, T., S. Pontinen y otros cuatro autores, Confronting the Technological Pedagogical Knowledge of Finnish Net Generation Student Teachers, doi: 10.1080/1475939X.2010.534867 Technology, Pedagogy and Education, 20(1), (2011) 